VOL. I3 (1975), 69-74.

\title{
On smoothness of the Banach space embedding
}

\section{J.R. Giles}

For a Banach space $X$, smoothness at a point of the natural embedding $\hat{X}$ in $X^{* *}$, is characterised by a continuity property of the support mapping from $X$ into $X^{*}$. It then becomes clear that there are many non-reflexive Banach spaces with smooth embedding, a matter of interest raised by I van Singer [Buzl. Austral. Math. Soc. 12 (1975), 407-416].

For a normed linear space $X$ with dual space $X^{*}$, to each $x \in S(X) \equiv\{x \in X:\|x\|=1\}$ we consider the set

$$
D(x) \equiv\left\{f \in S\left(X^{*}\right): f(x)=1\right\} ;
$$

(by the Hahn-Banach Theorem, $D(x)$ is non-empty). A support mapping $x \mapsto f_{x}$ of $X$ into $X^{*}$ maps each $x \in S(X)$ to $f_{x} \in D(x)$ and, for real $\lambda \geq 0, f_{\lambda x}=\lambda f_{x} . X$ is smooth at $x \in S(X)$ if $D(x)$ contains only one point. The norm of $X$ is weakly (Gâteaux) differentiable at $x \in S(X)$ if, for real $\lambda$,

$$
\lim _{\lambda \rightarrow 0} \frac{\|x+\lambda y\|-\|x\|}{\lambda} \text { exists for all } y \in S(X) \text {, }
$$

and is strongly (Fréchet) differentiable at $x \in S(X)$ if convergence to this limit is uniform for all $y \in S(X)$. $X$ is smooth at $x \in S(X)$ if and only if the norm is weakly differentiable at $x$ [3, p. 109].

We show the equivalence of two sets of conditions used to characterise differentiability of the norm. Our prof uses the extension to the Bishop-

Received 8 April 1975. 
Phelps Theorem given by Bollobás [1, p. 181].

THE BISHOP-PHELPS-BOLLOBÁS THEOREM. For a Banach space $X$, given $0<\varepsilon<1$ and $x \in S(X), f \in S\left(X^{*}\right)$ such that $|f(x)-1|<\varepsilon^{2} / 4$, there exists a $y \in S(X)$ and $f_{y} \in D(y)$ such that $\left\|f-f_{y}\right\|<\varepsilon$ and $\|x-y\|<\varepsilon$.

LEMMA. For a Banach space $X$, the following conditions are equivalent at $x \in S(X)$ :

1. for every sequence $\left\{f_{n}\right\}$ in $s\left(X^{*}\right)$ such that $f_{n}(x) \rightarrow 1$, we have that $\left\{f_{n}\right\}$ is

(i) $\sigma\left(X^{*}, X\right)-$,

(ii) $\sigma\left(X^{*}, X^{* *}\right)-$,

(iii) norm-convergent;

2. every support mapping $x \mapsto f_{x}$ of $X$ into $X^{*}$ is continuous on $S(X)$ at $x$ when $X$ has the norm-topology and $X^{*}$ has the

(i) $\sigma\left(X^{*}, X\right)-$,

(ii) $\sigma\left(X^{*}, X^{* *}\right)-$,

(iii) norm-topology.

Proof. Suppose that 1 holds. It is clear that all such sequences $\left\{f_{n}\right\}$ converge to the same limit $f$ where $\|f\| \leq I$. Then for any sequence $\left\{x_{n}\right\}$ in $S(X)$ where $\left\{x_{n}\right\}$ is norm-convergent to $x$, we have for $f_{x_{n}} \in D\left(x_{n}\right)$ that

$$
\left|f_{x_{n}}(x)-1\right|=\left|f_{x_{n}}(x)-f_{x_{n}}\left(x_{n}\right)\right| \leq\left\|x-x_{n}\right\| ;
$$

so $f_{x_{n}}(x) \rightarrow 1$ and, therefore, the sequence $\left\{f_{x_{n}}\right\}$ is
(i) $\sigma\left(X^{*}, X\right)-$,
(ii) $\sigma\left(X^{*}, X^{* *}\right)-$,
(iii) norm-convergent to $f$.

Since 


$$
\begin{aligned}
|f(x)-1| & \leq\left|f(x)-f_{x_{n}}(x)\right|+\left|f_{x_{n}}(x)-f_{x_{n}}\left(x_{n}\right)\right| \\
& \leq \mid\left\{f-f_{x_{n}}|(x)|+\left\|x-x_{n}\right\|,\right.
\end{aligned}
$$

we have that $f \in D(x)$. So there exists a support mapping which is continuous on $S(X)$ at $x$. However, this implies that $X$ is smooth at $x$ and that every support mapping is continuous at $x[3$, p. 107 and p. 109$]$.

Suppose that 2 holds. This implies that $X$ is smooth at $x$. For any sequence $\left\{f_{n}\right\}$ in $S\left(X^{*}\right)$ where $f_{n}(x) \rightarrow 1$, we have, by the BishopPhelps-Bollobás Theorem, that there exists a sequence $\left\{y_{n}\right\}$ in $S(X)$ and a sequence $\left\{f_{y_{n}}\right\}$ where $f_{y_{n}} \in D\left(y_{n}\right)$, such that $\left\{f_{n}-f_{y_{n}}\right\}$ is normconvergent to 0 and $\left\{y_{n}\right\}$ is norm-convergent to $x$. But then $\left\{f_{y_{n}}\right\}$ is

$$
\begin{aligned}
& \text { (i) } \sigma\left(X^{*}, X\right)-\text {, } \\
& \text { (ii) } \sigma\left(X^{*}, X^{* *}\right)- \\
& \text { (iii) norm-convergent to } f_{x} \in D(x) \text {. }
\end{aligned}
$$

Therefore, $\left\{f_{n}\right\}$ is

$$
\begin{aligned}
& \text { (i) } \sigma\left(X^{*}, X\right)-, \\
& \text { (ii) } \sigma\left(X^{*}, X^{* *}\right)-, \\
& \text { (iii) norm-convergent to } f_{x} \text {. }
\end{aligned}
$$

Smulian has shown the equivalence of 1 (i) to weak differentiability of the norm at $x[9, \mathrm{p}, 91]$ and 1 (iii) to strong differentiability of the norm at $x$ [10, p. 645]. The equivalence of 2 (i) to weak differentiability of the norm at $x$ and 2 (iii) to strong differentiability of the norm at $x$ is shown in [3, p. 107]. It is the particular equivalence of 1 (ii) and 2 (ii) which we require and it will follow from our theorem that these conditions are themselves equivalent to weak differentiability of the norm at $\hat{x} \in X^{* *}$. 
Our theorem relies essentially on the following result given by Lindenstrauss and Tzafriri [6, p. 196].

THE PRINCIPLE OF LOCAL REFLEXIVITY. For a Banach space $X, A$ is a finite dimensional subspace of $X^{* *}, B$ is a finite set in $X^{*}$, and $0<\delta<1$. There exists a linear mapping $T: A+X$ such that $T(\hat{x})=x$ for alz $\hat{x} \in A \cap \hat{X}, f(T(F))=F(f)$ for alz $F \in A$ and $f \in B$, and $(1-\delta)\|F\| \leq\|T(F)\| \leq(1+\delta)\|F\|$ for alz $F \in A$.

THEOREM. For a Banach space $X, X^{* *}$ is smooth at $\hat{x} \in S(\hat{X})$ if and only if every support mapping $x \mapsto f_{x}$ of $X$ into $X^{*}$ is continuous on $S(X)$ at $x$ when $X$ has the norm-topology and $X^{*}$ has the $\sigma\left(X^{*}, X^{* *}\right)$ topology.

Proof. Suppose that $X^{* *}$ is smooth at $\hat{x} \in S(\hat{X})$. Then every support mapping $F \mapsto \mathrm{F}_{F}$ of $X^{* *}$ into $X^{* * *}$ is continuous on $S\left(X^{* *}\right)$ at when $X^{* *}$ has the norm-topology and $X^{* * *}$ has the $\sigma\left(X^{* * *}, X^{* *}\right)-$ topology, [3, p. 107]. Every support mapping $x \mapsto f_{x}$ of $X$ into $X^{*}$ induces a support mapping $\hat{x} \mapsto \hat{f}_{x}$ of $\hat{X}$ into $\hat{X}^{*}$ which can be extended to a support mapping $F \mapsto F_{F}$ of $X^{* *}$ into $X^{* * *}$, and so it is continuous on $S(X)$ at $x$ when $X$ has the norm-topology and $X^{*}$ has the $\sigma\left(X^{*}, X^{* *}\right)$-topology.

Suppose that $X^{* *}$ is not smooth at $\hat{x} \in S(\hat{X})$; that is, there exists an $\hat{f}_{x} \in S\left(\hat{X}^{*}\right)$ and $F \in S\left(X^{* * *}\right)$ where $F \neq \hat{f}_{x}$ such that $f_{x}(x)=F(\hat{x})=1$. Now there exists an $F \in S\left(X^{* *}\right)$ and an $r>0$ such that $\left(F-\hat{f}_{x}\right)(F) \geq r$. Using the Principle of Local Reflexivity as in [11], we have that for every $\delta>0$ there exists a mapping $T^{\delta}: \operatorname{span}\left\{\hat{f}_{x}, \mathrm{~F}\right\} \rightarrow X^{*}$ such that $T^{\delta}\left(\hat{f}_{x}\right)=f_{x}, \hat{x}\left(T^{\delta} \mathrm{F}\right)=\mathrm{F}(\hat{x})=1$, $F\left(T^{\delta} \mathrm{F}-f_{x}\right)=\left(\mathrm{F}-\hat{f}_{x}\right)(F) \geq r$, and $1-\delta \leq\left\|T^{\delta} \mathrm{F}\right\| \leq 1+\delta$. Now consider a sequence $\left\{\delta_{n}\right\}$ where $\delta_{n} \rightarrow 0+$. The sequence $\left\{f_{n}\right\}$ in $S\left(X^{*}\right)$, where $f_{n}=\left(T^{\delta} n_{\mathrm{F}}\right) /\left(\left\|T^{\delta}{ }^{n} \mathrm{~F}\right\|\right)$, has the property that $f_{n}(x) \rightarrow 1$, but lim inf $F\left(f_{n}-f_{x}\right) \geq r$, so $\left\{f_{n}\right\}$ is not $\sigma\left(X^{*}, X^{* *}\right)$-convergent to $f_{x}$. It follows from the lemma that there exists a support mapping which is not 
continuous on $S(X)$ at $x$ when $X$ has the norm-topology and $X^{*}$ has the $\sigma\left(X^{*}, X^{* *}\right)$-topology.

We deduce, from the continuity characterisation of strong differentiability of the norm [3, p. 107], the following implication of our theorem.

COROLLARY 1. A Banach space $X$ with norm strongly differentiable at $x \in S(X)$ has $X^{* *}$ smooth at $\hat{x} \in S(\hat{X})$.

For a non-reflexive Banach space $X, X^{* * *}$ has a non-smooth point in $S\left(\hat{X}^{*}\right)$, [5]. In [8] Singer has shown that for a non-reflexive Banach space $X$ with a certain subspace property, $X^{* *}$ has a non-smooth point in $S(\hat{X})$. Our theorem provides us with many examples of non-reflexive Banach spaces with smooth embedding. Using Restrepo's result, [7], that every Banach space $X$ with separable dual $X^{*}$ can be equivalently renormed to have norm strongly differentiable on $S(X)$, we can specify a significant class of such spaces.

COROLLARY 2. A Banach space $X$ with separable dual $X^{*}$ oan be equivalently renormed to have $X^{* *}$ smooth on $S(\hat{X})$.

It is of interest to observe that a Banach space $X$ with separable dual $X^{*}$, so renormed does not necessarily have $X^{* *}$ smooth. If $c_{0}$ is so renormed, then $\hat{c}_{0}$ is smooth; but Day has shown that $m$ cannot be given an equivalent smooth norm [2, p. 522]. However, it is possible to have $c_{0}$ so renormed that $m$ is strongly differentiable on $D\left(z_{1}\right),[4$, p. 395].

Note added in proof (13 June 1975). Dr E.N. Dancer has pointed out that the theorem can also be proved using the fact that $\hat{B}\left(X^{*}\right)$ is $\sigma\left(X^{* * *}, X^{* *}\right)$-dense in $B\left(X^{* * *}\right)$ and without using the principle of local reflexivity.

\section{References}

[1] Béla Bollobás, "An extension to the theorem of Bishop and Phelps", Buzl. London Math. Soc. 2 (1970), 181-182. 
[2] Mahlon M. Day, "Strict convexity and smoothness of normed spaces", Trans. Amer. Math. Soc. 78 (1955), 516-528.

[3] J.R. Giles, "On a characterisation of differentiability of the norm of a normed linear space", J. Austral. Math. Soc. 12 (1971), 106-114.

[4] J.R. Giles, "On a differentiability condition for reflexivity of a Banach space", J. Austral. Math. Soc. 12 (1971), 393-396.

[5] J.R. Giles, "A non-reflexive Banach space has non-smooth third conjugate space", Canad. Math. Bulz. 17 (1974), 117-119.

[6] Joram Lindenstrauss and Lior Tzafriri, Classical Banach spaces (Lecture Notes in Mathematics, 338. Springer-Verlag, Berlin, Heidelberg, New York, 1973).

[1] Guillermo Restrepo, "Differentiable norms in Banach spaces", Bull. Amer. Math. Soc. 70 (1964), 413-414.

[8] Ivan Singer, "On the problem of non-smoothness of non-reflexive second conjugate spaces", Bull. Austral. Math. Soc. 12 (1975), $407-416$.

[9] В.Л. Шмульян [V.L. Smulian], "О неноторых геометричесннх свойствах единичной сферы нространства типа (B) " [On some geometrical properties of the unit sphere in the space of type (B) ], Mat. Sb. N.S. 48 (1938), 90-94.

[10] V.L. Šmulian, "Sur la dérivabilité de la norme dans l'espace de Banach", C.R. (DokZ.) Acad. Sci. URRS 27 (1940), 643-648.

[11] Francis Sullivan, "Some geometric properties of higher duals of Banach spaces", Proc. Conf. Radon-Nikodym Prop. Kent. State. (to appear).

Department of Mathematics,

University of Newcastle,

Newcastle,

New South wales. 